\title{
Investigation of Clusters and Their Effect on Grain Growth in Single Phase AlxCoCrFeNi High Entropy Alloys
}

\author{
Bharat Gwalani ${ }^{1}$, Riyadh Salloom ${ }^{1}$, Talukder Alam ${ }^{1}$, Sheena V. Grace ${ }^{1}$, Srivilliputhur Srinivasan ${ }^{1}$ and \\ Rajarshi Banerjee ${ }^{1}$ \\ 1. Department of Materials Science and Engineering, University of North Texas, Denton, TX.
}

Solid solution strengthening, within random solid solutions, is a fundamental strengthening mechanism in most alloy systems. While traditionally this strengthening mechanism was largely developed and applied to alloys with one principal component, this notion can be easily extended to high entropy alloys (HEAs). This new class of alloys typically consists of five or more principal components in near equi-atomic proportion. The high configurational entropy in these alloys, often results in the stabilization of single concentrated solid solution phases.

With increased Al, sluggish grain growth kinetics and unusually high activation energies for grain growth is demonstrated in $\mathrm{AlxCoCrFeNi}(\mathrm{x}=0.1 \& 0.3)$ after solution heat-treatment despite the absence of any secondary precipitates. The calculations were done by annealing the AlxCoCrFeNi alloys are three different temperatures $\left(950^{\circ} \mathrm{C}, 1075^{\circ} \mathrm{C}\right.$ and $\left.1150{ }^{\circ} \mathrm{C}\right)$ for various times and measuring the grain sizes $(\mathrm{d})$. The slope of $\mathrm{d}^{\mathrm{n}}$ vs time is plotted with inverse of temperature $(\mathrm{T})$ as shown in Figure 1 and apparent activation energy of grain growth of the three different alloy compositions are estimated.

While, astronomically high values of the apparent activation energy, such as $568 \mathrm{~kJ} / \mathrm{mol}$, are not expected in fcc-based metallic alloys, more detailed microstructural analysis using atom probe tomography reveals a solute clustering tendency developing with increasing Al content in this HEA. Such solute clustering can change grain growth from a solute drag based to a much more sluggish cluster drag based mechanism. Atom probe microscopy was utilized to study the clusters in the AlxCoCrFeNi.

Our study by the in-built tools of IVAS software package indicate that while $\mathrm{Al}_{0.1} \mathrm{CoCrFeNi}$ alloy was found homogeneous in a highly cold worked and recrystallized condition, $\mathrm{Al}_{0.3} \mathrm{CoCrFeNi}$ matrix contained Al-rich clusters (see figure 2). These clusters are proposed to act as sites for dislocation pinning, and subsequently provide hindrance to grain growth through 'cluster drag' effect. Their composition and morphology are measured and reported here. The raw data is treated with other custom-built statistical tools to gain more insight into the clustering phenomenon. 

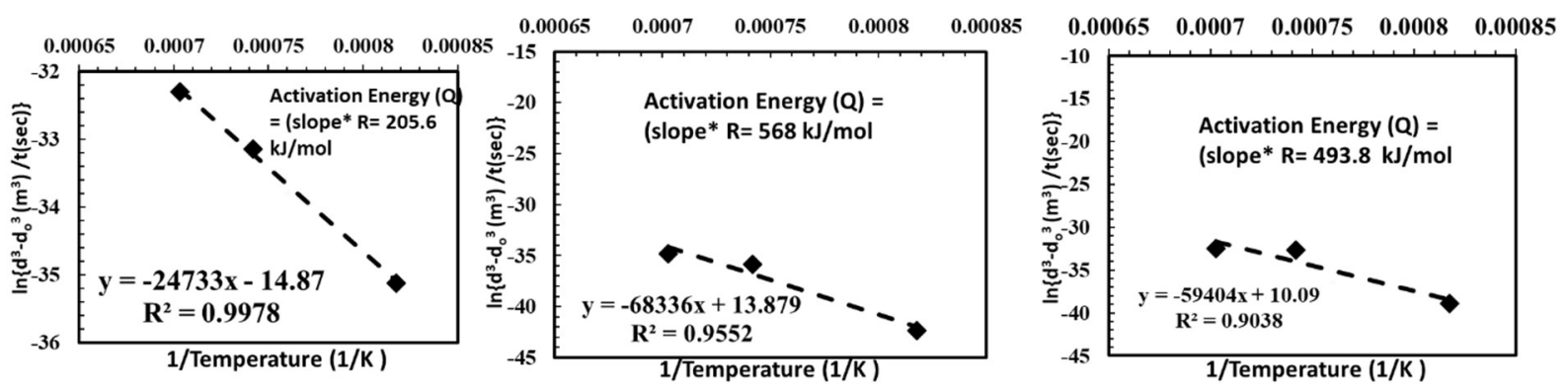

Figure 1. Activation energy for grain growth calculated for $\mathrm{Al}_{0.1} \mathrm{CoCrFeNi}, \mathrm{Al}_{0.2} \mathrm{CoCrFeNi}$ and $\mathrm{Al}_{0.3} \mathrm{CoCrFeNi}$.

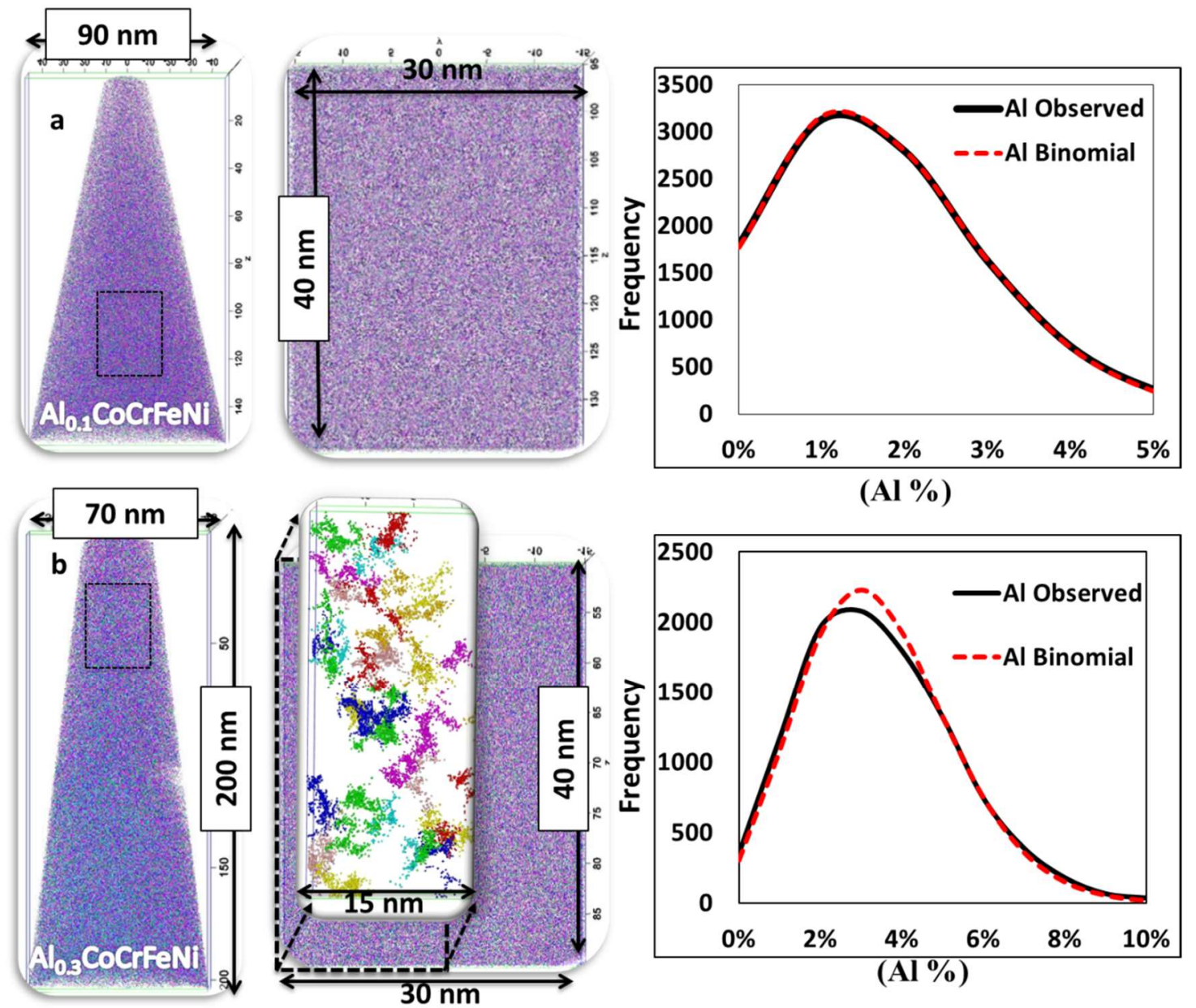

Figure 2. (a) atom probe reconstruction from $\mathrm{Al}_{0.1} \mathrm{CoCrFeNi}$ (top left image), a magnified view of the highlighted region from the reconstruction is shown in center and binomial and observed distribution of $\mathrm{Al}$ atoms in the highlighted region (top right image). (b) Atom probe reconstruction from $\mathrm{Al}_{0.3} \mathrm{CoCrFeNi}$ (bottom left image), a magnified view of highlighted region from the reconstruction (in center) and binomial and observed distribution of $\mathrm{Al}$ atoms in the selected region (bottom right). 